УДК 004.85

\title{
ПРОГНОЗИРОВАНИЕ КОТИРОВОК ЦЕННЫХ БУМАГ МЕТОДАМИ ЛИНЕЙНОЙ РЕГРЕССИИ, ДЕРЕВА РЕШЕНИЙ И С ПОМОЩЬЮ МНОГОСЛОЙНОЙ НЕЙРОННОЙ СЕТИ
}

\author{
Ким Наталья Георгиевна \\ Хлебородова Лидия Дмитриевна \\ студенты \\ Научный руководитель: Осипов Геннадий Сергеевич \\ д.т.н., зав. кафедрой Информатики \\ ФГБОУ ВО «Сахалинский государственный университет»
}

Аннотация: Разработан программный комплекс, позволяющий решать задачу прогнозирования котировок ценных бумаг на основании обучающей выборки, содержащей более тысячи наблюдений.

Апробировались и сравнивались методы линейной регрессии, дерева решений (decision tree) и искусственной нейронной сети.

Ключевые слова: прогноз котировок ценных бумаг, линейная регрессия, дерево решений, нейронная сеть.

\section{PREDICTING STOCK PRICES USING LINEAR REGRESSION, DECISION TREE, AND MULTI-LAYER NEURAL NETWORK}

\section{Kim Natalia Georgievna Khleborodova Lydia Dmitrievna Scientific adviser: Osipov Gennady Sergeevich}

\footnotetext{
Abstract: A software package has been developed that allows solving the problem of predicting securities quotes based on a training sample containing more than a thousand observations.

Linear regression, decision tree and artificial neural network methods were tested and compared.
}

Key words: stock price forecast, linear regression, decision tree, neural network. 


\section{1. Постановка задачи}

Объектом и предметом исследования является проблема построения функции $f$ регрессии (авторегрессии) вида [1]:

$$
f[\mathbf{x}(\tau), y(\tau)] \Rightarrow y(t+1) \mid t \geq 0
$$

где $\mathbf{x}(\cdot)$ - вектор внутренних аргументов;

$y(\cdot)$ - прогнозируемая величина.

Целью - отработка методологии синтеза искомой функции $f$ методом извлечения новых знаний из базы данных с помощью методов линейной регрессии, дерева решений и обучаемой многослойной искусственной нейронной сети.

В качестве исследовательского инструментария выбрана система символьной математики Wolfram Mathematica [2], которая является одной из наиболее продвинутых унифицированных платформ компьютерного моделирования, решения задач машинного обучения и разработок в области синтеза систем искусственного интеллекта.

\section{2. Обучающая выборка}

На рисунке 1 представлен оператор ввода импорта данных, а также начальный и конечный фрагменты обучающей выборки из 1003 записей.

\begin{tabular}{|c|c|c|c|c|c|c|c|c|}
\hline \multicolumn{9}{|c|}{ data = First @ Import ["Test.xlsx", "Dataset", "HeaderLines" $\rightarrow$ 1] } \\
\hline & Date & Open & High & Low & Close & Amount & Volume & Close+ \\
\hline & Fri 27 Jul 2018 00:00:00 & 243.99 & 244.74 & 241.06 & 243.25 & 2541960. & 617617305. & 243.73 \\
\hline & Sat $28 \mathrm{Jul} 2018$ 00:00:00 & 244.8 & 246.9 & 243.5 & 243.73 & 2966460. & 727341923. & 242.65 \\
\hline & Sun 29 Jul 2018 00:00:00 & 243.52 & 244.72 & 242.11 & 242.65 & 2712340. & 659072971. & 240.82 \\
\hline & Mon 30 Jul 2018 00:00:00 & 242.0 & 242.64 & 240.03 & 240.82 & 2103330. & 507203050. & 240.87 \\
\hline & Tue 31 Jul 2018 00:00:00 & 241.75 & 243.5 & 239.15 & 240.87 & 2594210. & 626355853. & 239.1 \\
\hline & Wed 21 Apr 2021 00:00:00 & 318.0 & 319.55 & 316.6 & 318.0 & 3446200. & 1097277610 & 315.9 \\
\hline & Thu 22 Apr 2021 00:00:00 & 319.0 & 319.85 & 310.75 & 315.9 & 6157270. & 1939588227. & 315.1 \\
\hline & Fri 23 Apr 2021 00:00:00 & 315.15 & 317.7 & 312.75 & 315.1 & 4402740. & 1388166557. & 318.5 \\
\hline & Sat 24 Apr 2021 00:00:00 & 314.75 & 318.5 & 313.8 & 318.5 & 2654440. & 839845251. & 317.85 \\
\hline
\end{tabular}

\section{Рис. 1. Фрагменты обучающей выборки}

В исследуемой задаче $\mathbf{x}=($ Open, High, Low, Close, Amount, Volume $)$, $y=($ Close, Close +$)-$ текущая и прогнозная цена закрытия. 


\section{3. Построение линейной регрессии}

На рисунке 2 приведен фрагмент текста в среде Wolfram Mathematica для обучения и тестирования (построения прогноза на требуемый отрезок времени) при использовании линейной регрессии

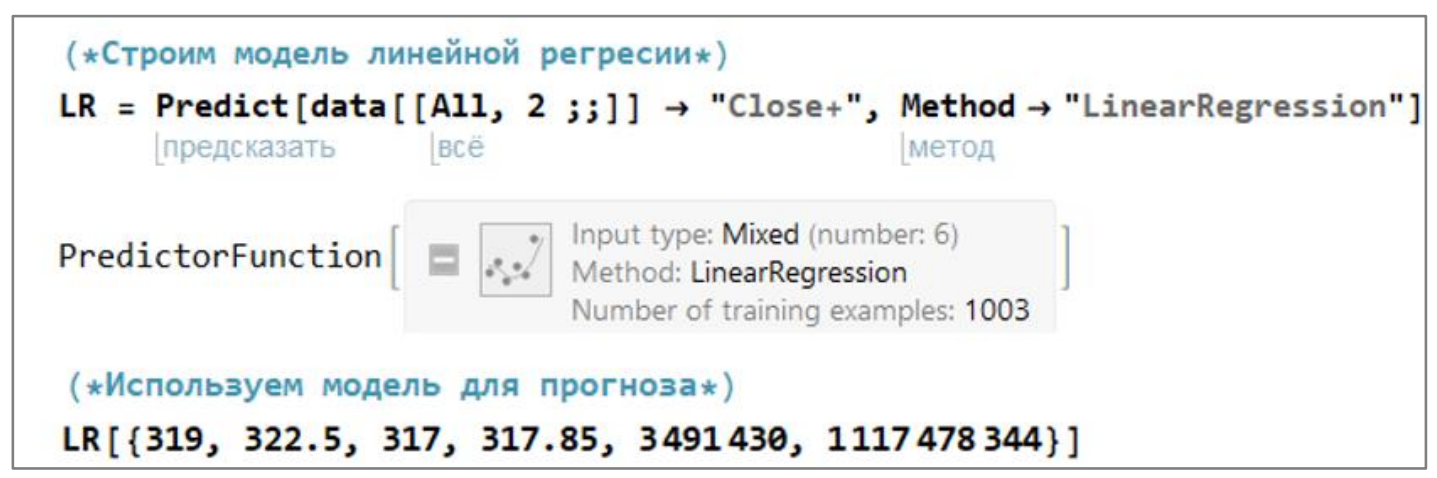

Рис. 2. Прогноз методом линейной регрессии

\section{4. Построение модели дерева решений}

На рисунке 3 приведены аналогичные по функционалу фрагменты текста при использовании в качестве метода предсказания метода дерева решений

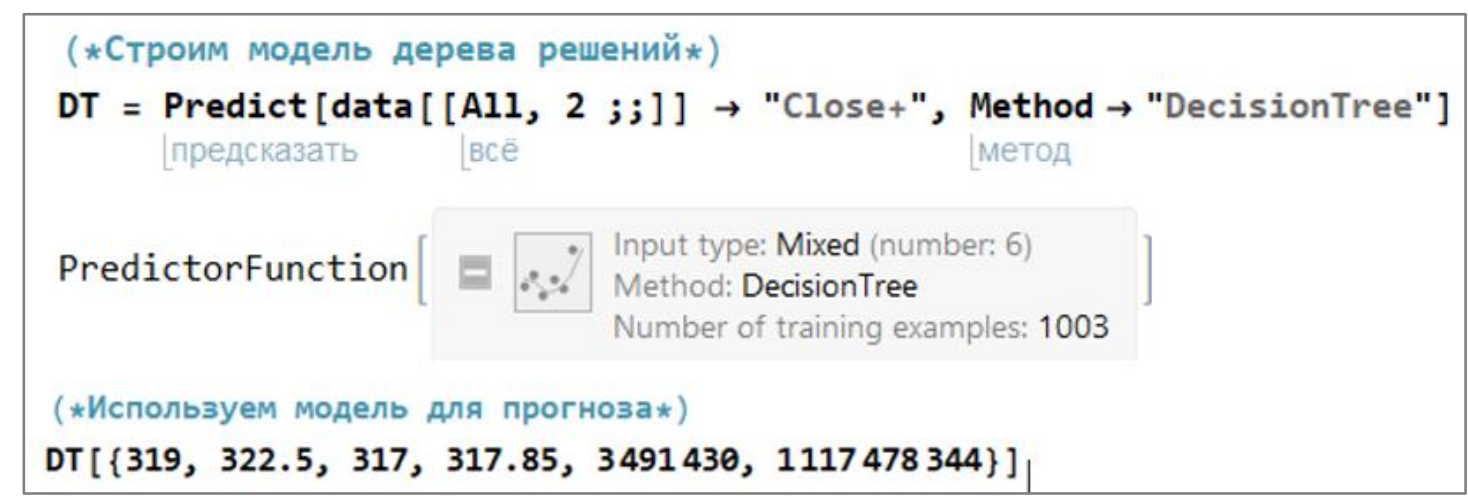

\section{Рис. 3. Прогноз методом дерева решений}

\section{5. Сравнение методов линейной регрессии и дерева решений}

Графическая интерпретация сравнения методов линейной регрессии и дерева решения при решении задачи интерполирования и прогнозирования приведена на рисунке 4. Для наглядности выведены только последние 250 (финальных) данных 


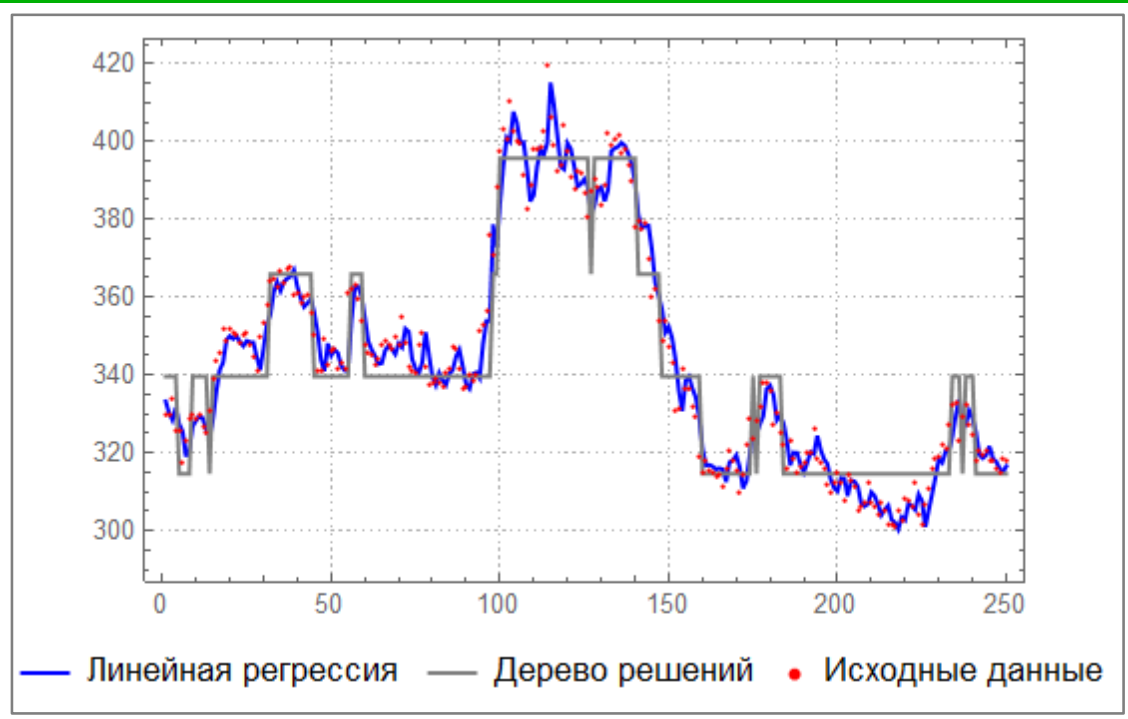

Рис. 4. Сравнение результатов обучения

\section{6. Использование нейронной сети}

За последнее время нейронные сети сети стали одной из базовых парадигм компьютерного моделирования и составляют основу синтеза ситем искусственного интеллекта.

В качестве примера на рисунке 5 представлен фрагмент текста по созданию в среде Wolfram Mathematica многослойной сети прямого распространения с шестью нейронами во входном слое (в соответствии с размерностью вектора внутренних аргументов $\mathbf{x}(\cdot))$ и одним нейроном в выходнос слое (в системе одна прогнозируемая величина Close +).

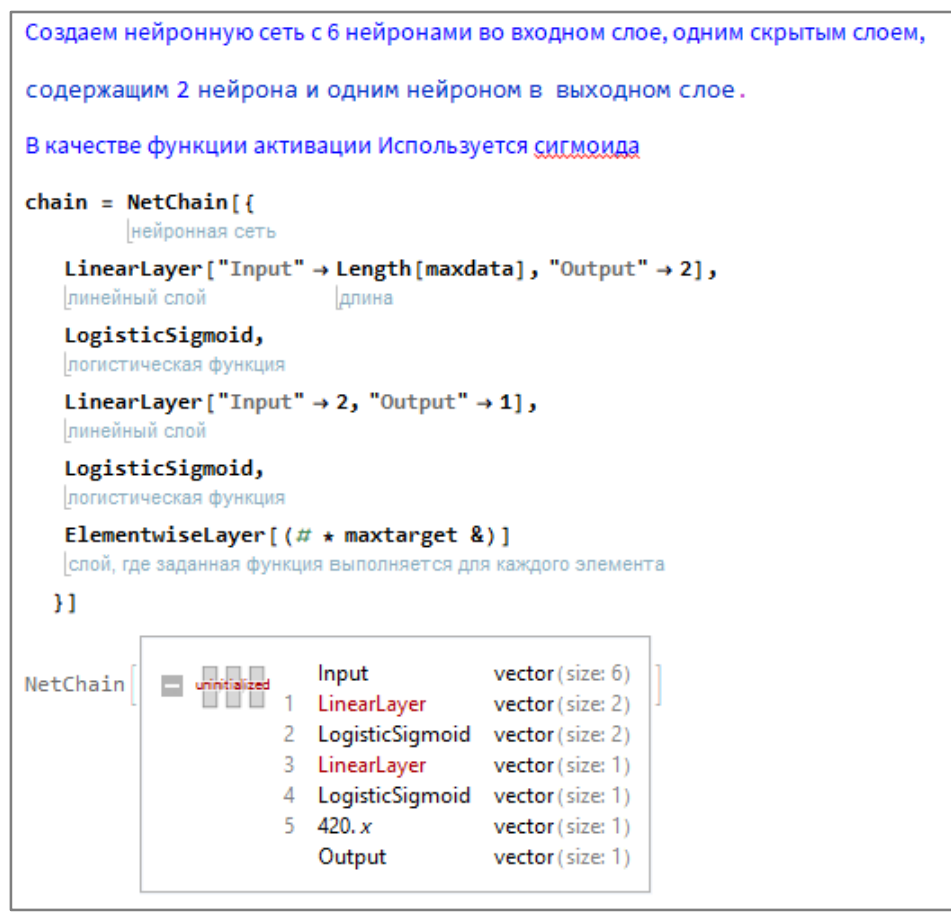

Рис. 5. Синтез нейронной сети 
Рисунок 6 демонстрирует текст и заключительный момент обучения нейронной сети с заданными основными параметрами - обучающей и тестовой выборкой, количеством эпох обучения.

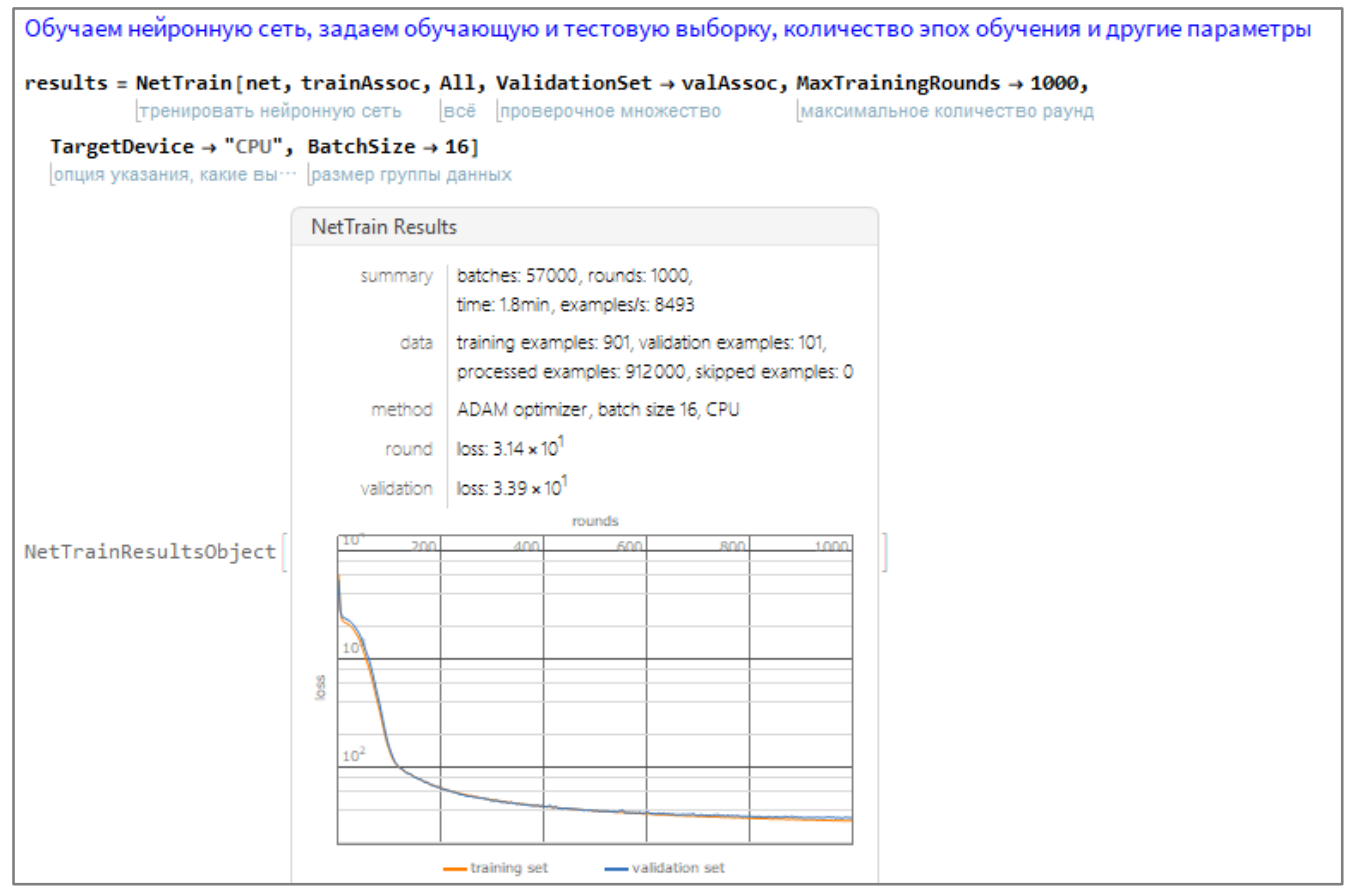

Рис. 6. Обучение нейронной сети

\section{Выводы}

Разработанное программмное обеспечение предназначено для исследования и прогнозирования комбинированных стохастических временных рядов, обладающих сезонными колебаниям, постепенным усвоением инсайдерской информации, подверженным финансовым пузырям, всзлетам и падениям.

Используемые методы машинного обучения позволяют получить прогноз с относительной ошибкой предсказания не более одного процента.

\section{Список литературы}

1. Осипов Г.С., Вашакидзе Н.С., Филиппова Г.В. Основы прогнозирования финансовых временных рядов на базе NeuroXL Predictor // Постулат. 2017. № 7. С. 26.

2. Stephen Wolfram. An Elementary Introduction to the Wolfram Language. URL: https://www.wolfram.com/language/elementary-introduction/2nd-ed/ (Дата обращения 07.05.2021).

(C) Н.Г. Ким, Л.Д. Хлебородова, Г.С. Осипов 2021 